\title{
Characterization of human myoblast cultures for tissue engineering
}

\author{
JENS STERN-STRAETER, GREGOR BRAN, FRANK RIEDEL, ALEXANDER SAUTER, \\ KARL HÖRMANN and ULRICH REINHART GOESSLER
}

\author{
Department of Otolaryngology, Head and Neck Surgery, University Hospital Mannheim, \\ University of Heidelberg, Mannheim, Germany
}

Received August 23, 2007; Accepted September 28, 2007

\begin{abstract}
Skeletal muscle tissue engineering, a promising specialty, aims at the reconstruction of skeletal muscle loss. In vitro tissue engineering attempts to achieve this goal by creating differentiated, functional muscle tissue through a process in which stem cells are extracted from the patient, e.g. by muscle biopsies, expanded and differentiated in a controlled environment, and subsequently re-implanted. A prerequisite for this undertaking is the ability to cultivate and differentiate human skeletal muscle cell cultures. Evidently, optimal culture conditions must be investigated for later clinical utilization. We therefore analysed the proliferation of human cells in different environments and evaluated the differentiation potential of different culture media. It was shown that human myoblasts have a higher rate of proliferation in the alamarBlue ${ }^{\circledR}$ assay when cultured on gelatin-coated culture flasks rather than polystyrene-coated flasks. We also demonstrated that myoblasts treated with a culture medium with a high concentration of growth factors [growth medium (GM)] showed a higher proliferation compared to cultures treated with a culture medium with lower amounts of growth factors [differentiation medium (DM)]. Differentiation of human myoblast cell cultures treated with GM and DM was analysed until day 16 and myogenesis was verified by expression of MyoD, myogenin, $\alpha$-sarcomeric actin and myosin heavy chain by semi-quantitative RT-PCR. Immunohistochemical staining for desmin, Myf-5 and $\alpha$-sarcomeric actin was performed to verify the myogenic phenotype of extracted satellite cells and to prove the maturation of cells. Cultures treated with DM showed positive staining for $\alpha$-sarcomeric actin. Notably, markers of differentiation were also detected in cultures treated with GM, but there was no formation of
\end{abstract}

Correspondence to: Dr Jens Stern-Straeter, Department of Otolaryngology, Head and Neck Surgery, University Hospital Mannheim, D-68135 Mannheim, Germany

E-mail: jens.stern-straeter@hno.ma.uni-heidelberg.de

Key words: tissue engineering, skeletal muscle, stem cells, cell culture, myoblasts, satellite cells myotubes. In the enzymatic assay of creatine phosphokinase, cultures treated with DM showed a higher activity, evidencing a higher degree of differentiation. In this study, we obtained detailed information regarding the cultivation and differentiation of human myoblast cultures in different environments. By exploring optimal culture conditions for skeletal muscle tissue engineering, we acquired culture data for comparison with other sources of stem cells in order to find the most applicable stem cell for focussed clinical usage.

\section{Introduction}

The functional restoration of skeletal muscle defects is a demanding process due to the difficulty in procuring donor tissue. Numerous muscle transfer techniques exist; however, all such approaches result in a certain donor-site morbidity, causing functional loss and volume deficiency. Skeletal muscle tissue engineering promises an alternative approach to generating functional muscle tissue.

The field of skeletal muscle tissue engineering has evolved to create efficient muscle tissue by using the regenerative potential of stem cells and their potential for proliferation and maturation. The preferred source of cells for skeletal muscle tissue engineering applications is primary, non-immunogenic satellite cells since they can easily be obtained by muscle biopsies and cultivated in vitro (1). Satellite cells from different species (e.g. the rat, mouse and chicken) can be isolated and have been studied extensively (2-5). In the last few years, various research groups have presented techniques for the generation of non-human vascularised three-dimensional skeletal muscle tissue using e.g. embryonic stem cells or the arteriovenous loop model, showing the enormous potential of this concept $(6,7$, reviewed in ref. 8). Human satellite cells can also be successfully extracted and expanded in vitro, meeting the requirements for future clinical applications (9). Nevertheless, the differentiation of human myoblast cell cultures under different conditions for the purpose of tissue engineering remains relatively unexplored. In order to investigate optimal culture conditions for human myoblast cell cultures and gain more insight into the maturation from myoblasts to myofibers, we analysed the cell cultures under different culture conditions and studied the implications of these conditions on cell proliferation and myogenesis.

Using the alamarBlue proliferation assay we compared the cell divisions of human myoblasts on both gelatin-coated 
culture flasks and non-coated polystyrene culture flasks. Having found higher rates of proliferation within the group of gelatin-coated cultures, we performed all further experiments on gelatin-coated culture flasks.

Next, we compared the proliferation and maturation of cells in two different culture media [growth medium (GM) and differentiation medium (DM)]. We analysed the maturation of mononucleated myoblasts into multinucleated myotubes by investigating the expression of marker genes that play well-defined roles during myogenesis. Among these genes, myogenic regulator factors (MRF) play an elemental role. They are transcription factors with a basic helix-loop-helix DNA-binding domain that binds to the specific DNA sequence CANNTG. They serve as promoters of several musclespecific genes that control the fusion of myoblasts to multinucleated myofibers (10). Myf-5, MyoD and myogenin are determining members of the MRF family (11).

Myf-5 and MyoD act as myogenic determination factors. MyoD is up-regulated in the early stages of myogenesis and induces both the fusion of myoblasts and the reduction of proliferation. This is accomplished through the activation of cell cycle inhibitors (p21) and the down-regulation of cell cycle activators such as cyclins and cyclin-dependent kinases (12). Myogenin arises in later stages of differentiation and acts more specifically in the formation of multinucleated muscle tissue (13). Myogenin-deficient knock-out mice terminate muscle formation on account of insufficient myoblast fusion (14). MyoD and myogenin are up-regulated during different periods of the skeletal muscle formation process and can therefore function as markers of differentiation (15). The final stage of differentiation can be identified by the expression of the structural proteins $\alpha$-sarcomeric actin and myosin heavy chain (MHC) as a part of the contractile apparatus. Therefore, we investigated the expression of Myf-5, MyoD, myogenin, $\alpha$-sarcomeric actin and MHC on days $0,4,8$ and 16 . Multinucleated myotubes could only be detected in cultures treated with DM, and these were positive for $\alpha$-sarcomeric actin immunohistochemical staining. We also assessed cell differentiation by measuring creatine kinase activity. These measurements revealed higher activity in cultures treated with DM.

\section{Materials and methods}

Cell culture. Human skeletal muscle biopsies from 15 patients were obtained during head and neck surgery. The median age of these patients was 58, ranging from 41 to 72 . The study was approved by the Ethics Committee of the Faculty of Medicine Mannheim, University of Heidelberg, Germany, and the patients gave informed consent. Satellite cells were dissociated from the minced muscles by digestion with collagenase B (Roche, Mannheim, Germany) for $60 \mathrm{~min}$ and $0.05 \%$ trypsin- $0.02 \%$ EDTA (PromoCell, Heidelberg, Germany) for $45 \mathrm{~min}$ at $37^{\circ} \mathrm{C}$. These were filtered through a sterile $70-\mu \mathrm{m}$ cell strainer (Becton Dickinson, Franklin Lakes, NJ, USA) and purified with the pre-plating technique described in (16). Purity of myoblast cultures ( $>80 \%$ ) was evaluated by anti-desmin immunostainings. Cells were grown on $0.2 \%$ gelatin-coated culture flasks (Sigma, Deisenhofen, Germany) and in Ham's F10 growth medium, containing 1\% penicillin/streptomycin/fungizone-solution (PSF), 2 mM Lglutamine (all from PromoCell) and $10 \%$ foetal bovine serum (FBS) (PAA Laboratories, Linz, Austria) (GM). Cells were maintained at $37^{\circ} \mathrm{C}$ in a humidified atmosphere of $5 \% \mathrm{CO}_{2}$ and $95 \%$ air, and the medium was changed every $72 \mathrm{~h}$.

Differentiation of skeletal muscle myoblasts. Skeletal myoblasts were cultured to $\sim 60 \%$ confluence, washed with phosphate-buffered saline (PBS) and induced to differentiate by a change in medium consisting of minimal essential medium (MEM) (PromoCell) supplemented with $2 \%$ horse serum (PAA), 2 mM L-glutamine and PSF (both from PromoCell) (DM).

AlamarBlue proliferation assay. Myoblasts were plated in 96-well culture plates coated with or without $0.2 \%$ gelatin at a density of 5000 cells/well in the growth medium. Proliferation was assessed over time on days 1, 2, 3, 4, 7, 8, 9, 10 and 11 . In the second experiment, cells were allowed to adhere for $24 \mathrm{~h}$, after which a switch to a fresh growth medium or differentiation medium took place. The alamarBlue assay was performed on days $0,4,8$ and 16 . The culture plates were incubated with the alamarBlue solution for $24 \mathrm{~h}$ before measurements of exiting fluorescence at a wavelength of $540 \mathrm{~nm}$. Absorbance was monitored at $590 \mathrm{~nm}$.

Immunohistochemistry. Immunohistochemical characterization was carried out on cells grown on chamber culture slides (BD Falcon, Franklin Lakes, NJ, USA). Myoblasts were identified using antibodies to desmin (Dako, Hamburg, Germany) and Myf-5 (Santa Cruz Biotechnology, Heidelberg, Germany), and maturation was evaluated using $\alpha$-sarcomeric actin as a differentiation marker (Zymed Laboratories, Invitrogen, Karlsruhe, Germany). The desmin-antibody was used in a solution of 1:100 and $\alpha$-sarcomeric actin at a dilution of 1:50, followed by a specific biotinylated secondary antibody and a streptavidin-biotin horseradish peroxidase complex. The peroxidase reaction was performed using aminoethylcarbazol (Dako) as chromogen. Endogenous peroxidase was blocked with $0.3 \%$ hydrogen peroxide for $30 \mathrm{~min}$. Sections were washed with PBS and incubated with normal sheep serum in PBS for $30 \mathrm{~min}$ at room temperature to block non-specific antibody reaction. Nuclei were counterstained with Harris' haematoxylin. Light microscopy investigation was performed using a Zeiss Axiophot microscope.

Creatine kinase analysis. Differentiation of myoblasts was identified by measuring the creatine phosphokinase activity of developing myofibers using the creatine phosphokinase assay (Sigma). Cells were lysated with a buffer containing $\mathrm{NP}-40$ and protease inhibitors and stored at $-80^{\circ} \mathrm{C}$. Samples were assayed according to the manufacturer's protocol. Enzymatic activity was normalized over the total protein content determined by the Lowry protein assay (Bio-Rad, Munich, Germany).

RNA isolation, cDNA synthesis and PCR. Total RNA was isolated with the RNA Mini Kit (Qiagen, Hilden, Germany), according to the manufacturer's instructions. Total RNA 
Table I. Oligonucleotide primers for polymerase chain reaction.

\begin{tabular}{|c|c|c|}
\hline Primer & Fragment size (bp) & $\begin{array}{ll}\text { Sequence } & \text { 1) } 5^{\prime}-\text { primer } 1-3^{\prime} \\
& \text { 2) } 5^{\prime} \text {-primer } 2-3^{\prime}\end{array}$ \\
\hline Desmin & 464 & $\begin{array}{l}\text { 1) 5'-TCT GAA GCT GAG TGG TA-3' } \\
\text { 2) 5'-CTT CTT GGT ATG GAC CTC AG-3' }\end{array}$ \\
\hline GAPDH & 178 & $\begin{array}{l}\text { 1) 5'-GGT GGA GGT CGG AGT CAA CGG A-3' } \\
\text { 2) 5'-GAG GGA TCT CGC TCC TGG AGG A-3' }\end{array}$ \\
\hline Myf-5 & 278 & $\begin{array}{l}\text { 1) 5'-AGA GAG CAG CTG GAG AAC TA-3' } \\
\text { 2) 5'-AGA GAG AGA AGC CAG ATC CT-3' }\end{array}$ \\
\hline MyoD & 500 & $\begin{array}{l}\text { 1) 5'-AAG CGC CAT CTC TTG AGG TA-3' } \\
\text { 2) 5'-GCG CCT TTA TTT TGA CC-3' }\end{array}$ \\
\hline Myogenin & 460 & $\begin{array}{l}\text { 1) 5'-CAG TGG AAG ATG TT CTG TG-3' } \\
\text { 2) 5'-GAA GAG ACC AGA ACA GGA GA-3' }\end{array}$ \\
\hline$\alpha$-sarcomeric actin & 196 & $\begin{array}{l}\text { 1) 5'-TCG CGG TCT CCC TGT CCT TG-3' } \\
\text { 2) 5'-CCG AGC CGG CTC CCT CTG CG-3' }\end{array}$ \\
\hline MHC & 750 & $\begin{array}{l}\text { 1) 5'-TGT GAA TGC CAA ATG TGC TT-3' } \\
\text { 2) 5'-GTG GAG CTG GGT ATC CTT GA-3' }\end{array}$ \\
\hline
\end{tabular}

concentration was determined by $\mathrm{A}_{260}$ and $\mathrm{A}_{280}\left(\mathrm{~A}_{260} / \mathrm{A}_{280}=\right.$ 1.7-2.0) measurements using an Ultraspec $1000 \mathrm{UV} /$ Visible Spectrophotometer (Amersham Pharmacia Biotech, Buckinghamshire, UK). Aliquots of $5 \mu \mathrm{g}$ total RNA per experimental group were pooled and reverse-transcribed using an oligo(dT)-primed first-strand cDNA synthesis kit (Roche), according to the manufacturer's recommendations.

All cDNA probes were analysed for desmin, Myf-5, MyoD, myogenin, $\alpha$-sarcomeric actin, MHC and GAPDH in a 30-cycle standard PCR, using Taq DNA polymerase (Amersham) and 2-5 $\mu 1 \mathrm{RT}$ products as templates, in a Primus 96 Plus Thermal Cycler (MWG Biotech). Primer sequences are shown in Table I. Electrophoresis was carried out in $2 \%$ agarose gels containing ethidium bromide. Products were visualized and photographed under UV light. Relative gene expression was quantified using the densitometric scanning software AIDA (Raytest, Straubenhardt, Germany) using GAPDH as the internal standard.

\section{Results}

AlamarBlue proliferation assay of human myoblasts on gelatin-coated culture flasks compared to non-coated polystyrene flasks. The fluorescent units (FU), assessed with $5000 /$ cells per well, were higher in satellite cell cultures grown on gelatin-coated cultures flasks than in those grown on non-coated polystyrene culture flasks at all investigated time points (Fig. 1). On day 1 of cell culture, the FU in cultures grown on gelatin-coated flasks were $18.3 \pm 0.19$, compared to $14.0 \pm 0.71$ in the cultures grown on non-coated polystyrene culture flasks. Until day 4, the FU increased steadily in both groups. Within the group on gelatin-coated flasks, the FU rose from $20.4 \pm 0.50$ on day 2 , to $22.8 \pm 0.47$ on day 3 , and $23.8 \pm 1.56$ on day 4 . We observed a corresponding increase to $16.5 \pm 0.29$ on day $2,17.9 \pm 0.47$ on day 3 , and $18.04 \pm 1.04$ on day 4 in the non-coated culture group. Between day 4 and day 7, both groups displayed the greatest augmentation of FU: $33.5 \pm 1.03$ in cultures grown on gelatin-coated flasks compared to $25.2 \pm 1.04$ within the non-coated group. Noncoated cultures remained relatively static after day 7 and showed only slight enhancement until day 11 . Over these days, the FU for the non-coated cultures measured 20.1 \pm 1.36 on day $9,26.6 \pm 0.73$ on day 10 and $27.1 \pm 0.19$ on day 11 . In contrast, after day 7 , gelatin-coated cultures presented higher concentrations of FU than non-coated cultures: $35.8 \pm 2.1$ on day $8,31.7 \pm 1.81$ on day $9,36.8 \pm 1.81$ on day 10 and $32.9 \pm 0.72$ on day 11 .

AlamarBlue proliferation assay of human myoblasts on gelatin-coated culture flasks treated with GM and DM. The FU from cultures treated with DM were at all times lower than those from GM-treated cultures, starting from 13.4 \pm 0.48 on day 1 and increasing to $21.8 \pm 0.44$ on day $4,25.5 \pm 1.60$ on day 8 and $29.9 \pm 2.38$ on day 12 , with a maximum augmentation of $48.8 \pm 1.45$ on day 16. GM-treated cultures started at $29.9 \pm 0.48$ on day 1 and grew increasingly, except for a slight drop on day 8 , until day 16 . The values obtained were $51.1 \pm 5.53$ for day $4,45.84 \pm 2.91$ on day $8,60.8 \pm 4.49$ on day 12 and $75.1 \pm 1.14$ for day 16 (Fig. 2).

RT-PCR analysis. Desmin and Myf-5 expression were detected in all analysed samples and verified the myogenic phenotype of all investigated cell cultures. Semi-quantitative RT-PCR of MyoD, normalized by GAPDH, detected higher amounts of mRNA in cell cultures treated with DM than in cultures treated with GM. Cultures treated with DM showed their highest expression on day 8 , followed by a reduction in MyoD mRNA concentration until day 16. GM-nourished 


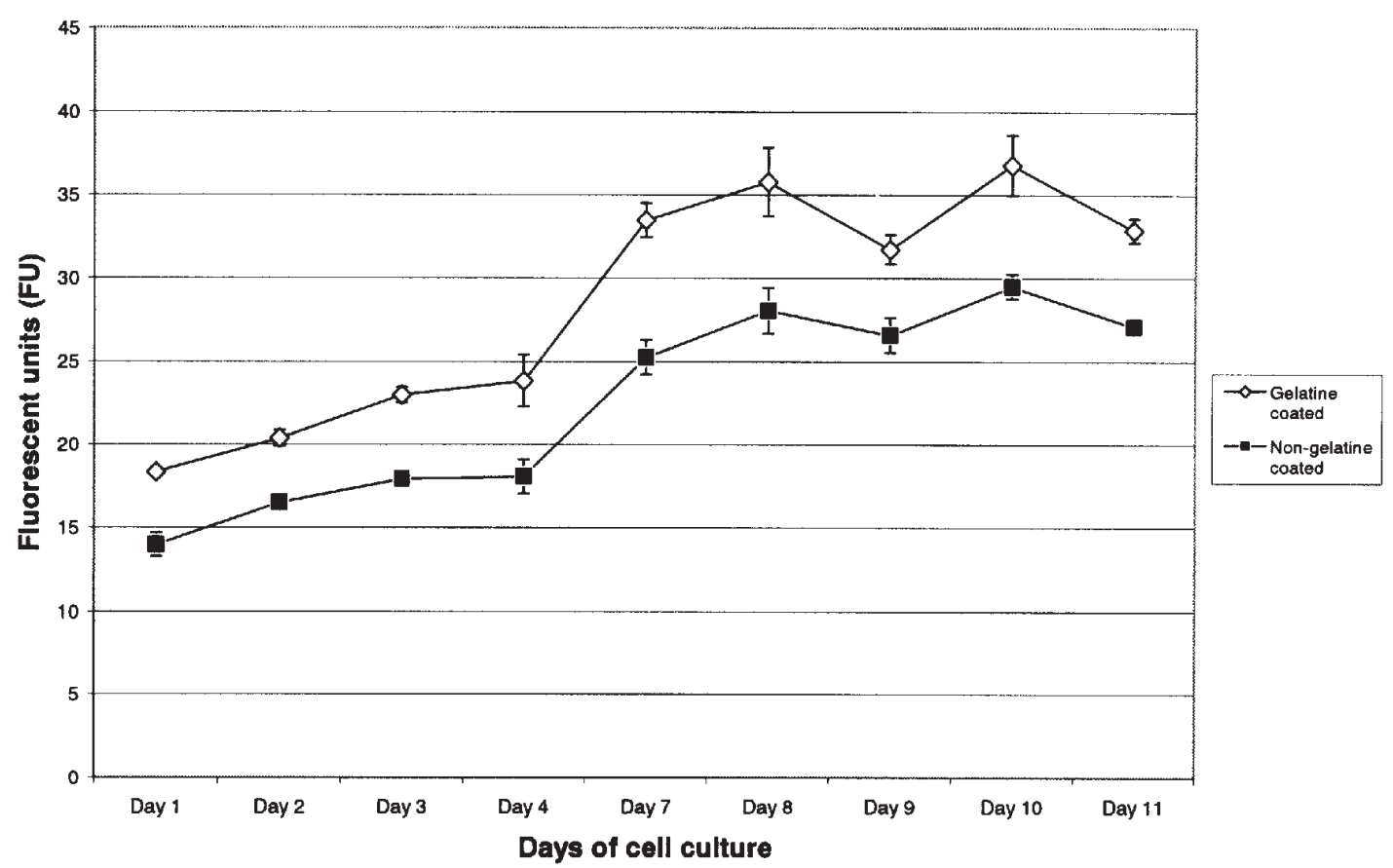

Figure 1. AlamarBlue proliferation assay of human myoblasts on gelatin-coated culture flasks compared to non-coated polystyrene flasks.

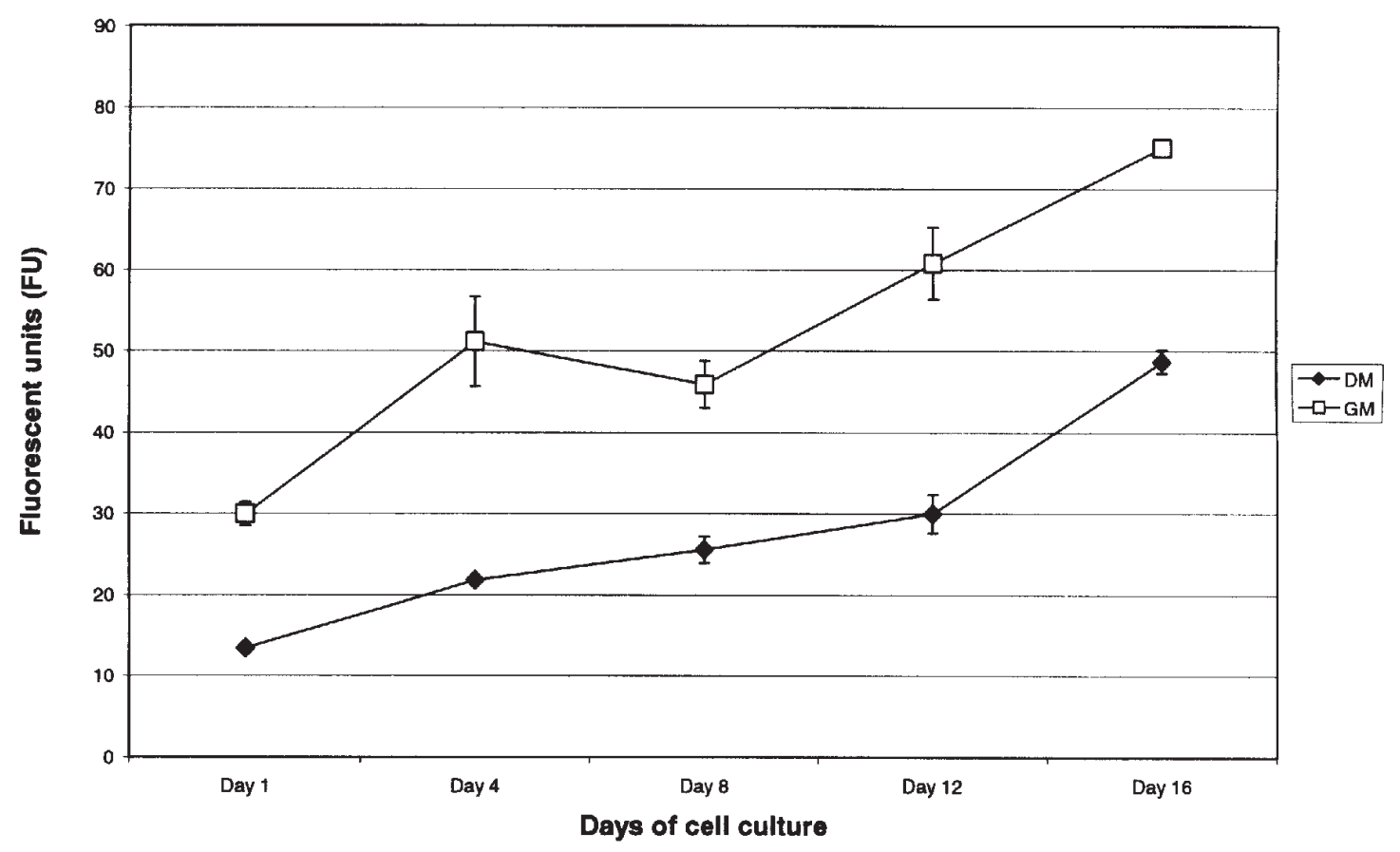

Figure 2. AlamarBlue proliferation assay of human myoblasts on gelatin-coated culture flasks treated with GM and DM.

cultures, on the other hand, displayed a slight increase in MyoD expression until day 16 (Fig. 3).

mRNA levels of myogenin were quantified in both study groups at all times and showed a steady increase from day 4 until day 16 in both groups.

Transcripts of $\alpha$-sarcomeric actin were detected in all analysed samples of cultures treated both with DM and with GM. Cultures treated with DM revealed a constant increase in the expression of $\alpha$-sarcomeric actin until day 12. Their highest expression occurred on day 16. GM-treated cultures, on the other hand, showed higher expression in the beginning of the experiment, declining on both days 12 and 16 .

Expression analysis of MHC proved constant augmentation from days 2 through 16 in both investigated groups.

Immunohistochemistry. In order to confirm that the primary cells utilized were of muscle origin and did not change phenotype during cultivation, cell cultures were investigated 


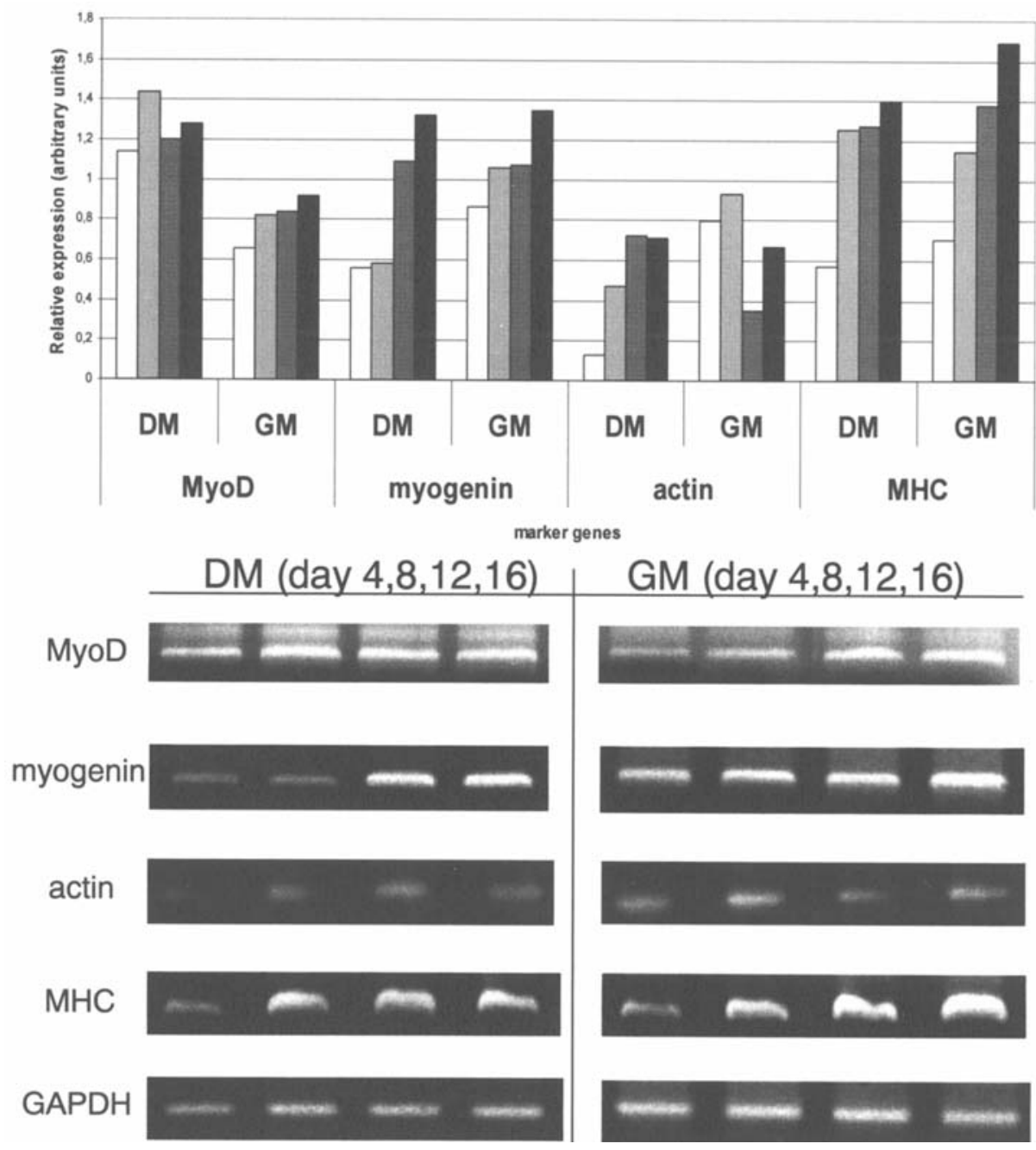

Figure 3. Densitometric quantification of MyoD, myogenin, $\alpha$-sarcomeric actin and MHC in human myoblast cultures during treatment with DM and GM using GAPDH as the internal standard.

by immunohistochemistry with using specific monoclonal antibodies against desmin, Myf-5 and $\alpha$-sarcomeric actin. All examined cultures were positive for the intermediary filament desmin and Myf-5, verifying the myogenic phenotype of assessed cells. After 8 days, $\alpha$-sarcomeric actin could be detected in myotubes in cell cultures treated with DM. It was never detected in cultures treated with GM (Fig. 4).

Creatine kinase $(C K)$ assay. To compare myogenesis quantitatively under different culture conditions, CK activity was measured in human myoblast cultures treated with GM and DM. As shown in Fig. 5, when cultured in DM, the specific activity of CK increased constantly after day 4 and was higher at the end of analysis than in GM-treated cultures. The activity of cultures treated with GM also increased until day 12 , but started to fall on day 16 . Therefore, our work reveals that more activity was stimulated in cultures treated with DM than in those which contained GM.

\section{Discussion}

Characterization of primary human myoblast cultures, and thereby an understanding of the process of differentiation from mononucleated myoblasts to myofibers, might lead to the clinical usage of skeletal muscle tissue engineering by revealing both the source of most the promising stem cells and the optimal culture conditions for each cell type. By applying the specific characteristics of various precursor cells, scaffolds and bioactive factors in order to form, manipulate and restore the skeletal tissue phenotype, it might be possible to regenerate muscle function for functional and aesthetic treatments.

In the last few years, different research groups have presented techniques for engineering various threedimensional skeletal muscle tissues that were able to produce force through contractions of muscle fibers (17). This engineered muscle cannot be compared to native muscle tissue, but it presents a major step forward. Other reports followed these important findings, introducing methods to vascularize engineered constructs in vitro $(6,7,18,19)$. However, these results were obtained with tumour-derived immortal cell lines like C2C12 (mouse) or L6 (rat), or xenogenic cell culture models which are merely experimental approaches that cannot be applied in a clinical scenario. Therefore, our study focussed on the characterization and optimization of human myoblast cell cultures in order to generate foundational information regarding proliferation rates during the maturation and expression pattern of 

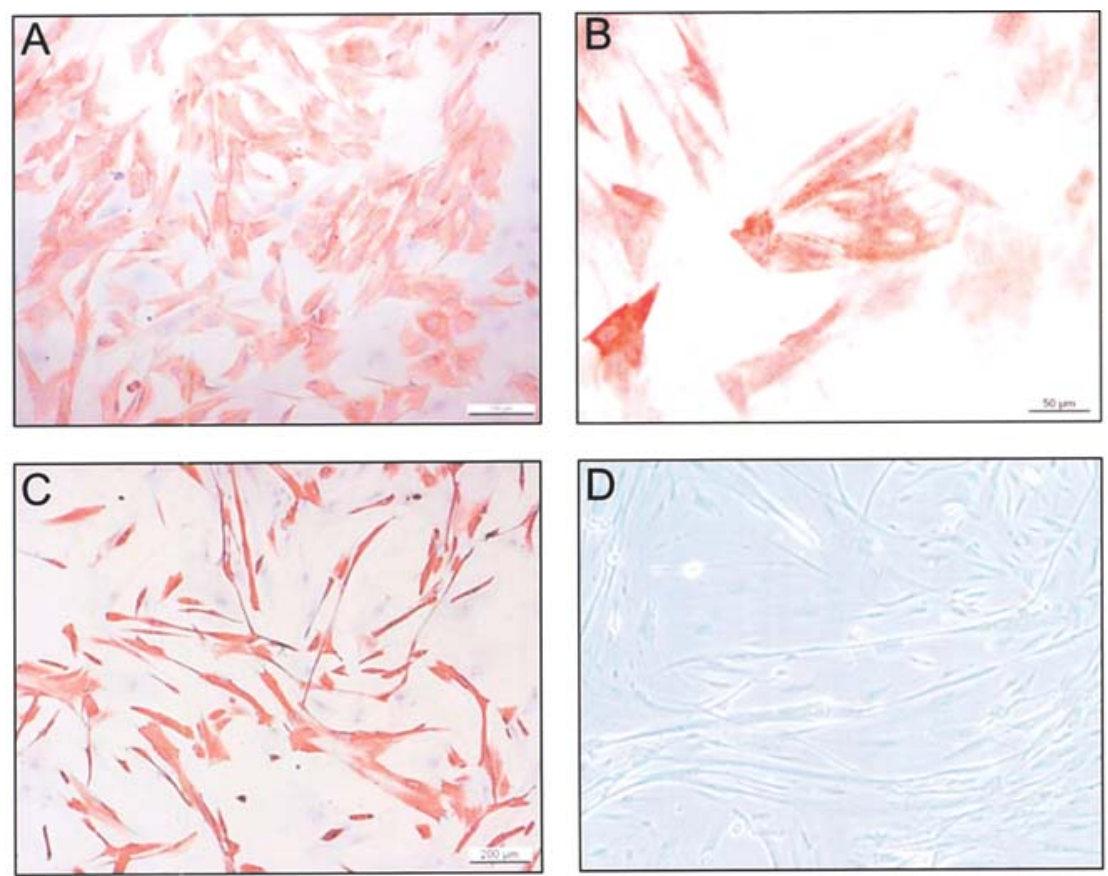

Figure 4. Immunohistochemical characterization and phase contrast light microscopy of human myoblast cultures. (A) Anti-desmin immunohistochemical staining of extracted cells verifying the purity of the myoblast cultures. (B) Anti-Myf-5 immunohistochemical staining of myoblasts on day 2 treated with GM. (C) Anti- $\alpha$-sarcomeric actin immunohistochemical staining of myotubes in cultures treated with DM on day 8. (D) Phase contrast light microscopy of human myoblasts on day 8 treated with DM.

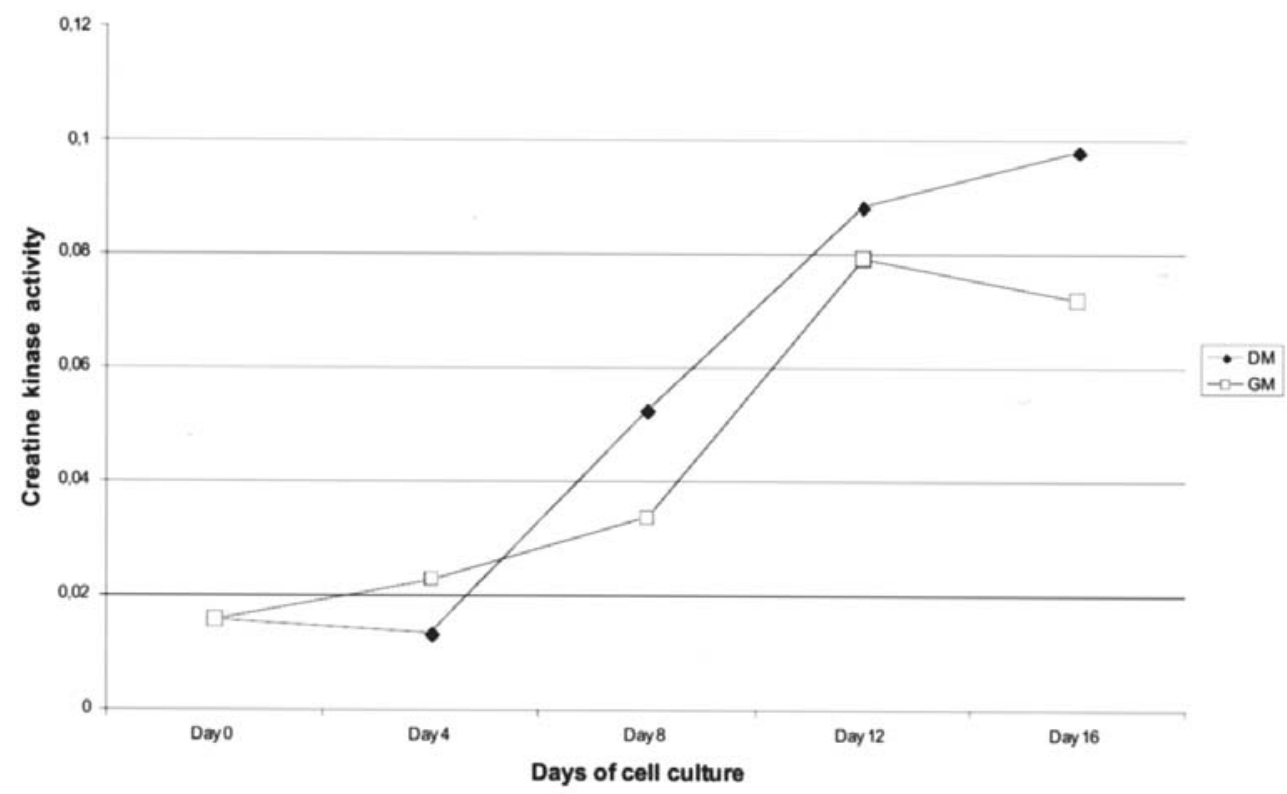

Figure 5. Creatine kinase activity assay of human myoblast cultures treated with GM and DM.

myogenesis for a later comparison with studies of other stem cells, like mesenchymal stem cells and human myoblasts cultured in a three-dimensional environment. The data obtained from the multi-potential human satellite cells can be accurately assigned to the tissue engineering concept. Therefore these findings are of particular importance for projects related to clinical research.

The surface morphology of the surrounding tissue plays an important role in the attachment, proliferation and differentiation of human satellite cells. Several reports have demonstrated that the composition of the extracellular matrix (ECM) proteins affect the alignment, proliferation and maturation of satellite cells $(20,21)$. For an accurate characterization of the obtained human myoblast cell pool, we measured the proliferation rate both with and without the use of ECM proteins, in order to deduce optimal culture conditions and evaluate the effects of the ECM on proliferation. We therefore analysed the cell division of primary human myoblasts on both gelatin-coated and noncoated polystyrene culture flasks using the alamarBlue assay. 
We confirmed the assertion of other groups that human myoblasts grown on gelatin dishes show higher rates of proliferation than non-coated dishes at all investigated times and were able to form myotubes under appropriate culture conditions (22). As a result, we performed all further experiments regarding the differentiation of myoblasts on gelatin-coated flasks.

Myogenesis of skeletal muscle is a highly complex phenomenon controlled and regulated by multiple intracellular signalling mechanisms (11). During skeletal muscle differentiation, mononucleated proliferating myoblasts stop dividing, activate muscle-specific gene expression and fuse into multinucleated myotubes. The apparent antagonism between proliferation and differentiation implies that the signalling pathways driving proliferation must be suppressed to allow for the induction of differentiation. This process depends on the growth factor activity. In cell culture, the differentiation of muscle cells can be induced by reducing the growth factor concentration in the culture media. In order to analyse the differences in proliferation between culture media containing high amounts (GM) and low amounts (DM) of growth factors, we performed the alamarBlue assay with GM with $10 \%$ foetal bovine serum and DM with $2 \%$ horse serum. We verified that, at all investigated time points, the proliferation rate of human myoblasts was higher in cultures treated with high amounts of serum (GM). However, the proliferation also increased gradually over time in cultures treated with DM, implying that some cells did not withdraw from the cell cycle and were induced to differentiate.

Since skeletal muscle has become a general model for understanding many fundamental principles of development, including the antagonism between growth and differentiation, we analysed the expression of marker genes which correspond with each event in the development from mononucleated myoblasts to multinucleated muscle fibers in cultures with higher proliferation rates treated with GM and cultures with a low serum content in order to induce the differentiation process. The factors that play a major role in controlling the events leading to skeletal muscle development are MyoD, Myf-5 and myogenin. By analysing the expression of the early myogenic transcription factor MyoD in cell cultures treated with DM and GM, we demonstrated that, in both experimental groups, cells were induced to differentiate. The densitometric evaluation revealed higher amounts of mRNA in cultures treated with DM, verifying the enhanced stimulation of myogenesis within the group treated with the serum-reduced media. Interestingly, MyoD was also expressed in cultures treated with GM, implying that slight variations in MyoD expression may change the balance between proliferation and differentiation (23). Analysing the expression of myogenin, another myogenic transcription factor of the MRF family known to be expressed in the later stages of myogenesis, we showed a time-dependent augmentation in the mRNA concentration in both groups, revealing the differentiation of cells in both study groups. The structure proteins $\alpha$-sarcomeric actin and MHC were also expressed in both study groups. Immunohistochemical evaluation of the myoblast cultures revealed that $\alpha$-sarcomeric actin-positive myotubes were detected only in cultures treated with DM.
These data demonstrate that the investigation of marker gene expression alone appears to be insufficient for an accurate characterization of myogenesis, since myotubes were only detected in cultures with a low serum content.

In order to quantify muscle differentiation more accurately, we measured creatine content and were able to show enhanced activity in cultures treated with DM, with a maximum at day 16.

In order to investigate optimal culture conditions and characterization of human myoblast cell cultures, we analysed cell behaviour under different culture conditions and showed that cultures treated with DM expressed several characteristic features of mature skeletal muscle. Also, in GM-treated cultures certain markers of differentiation were detected, demonstrating that, for an accurate characterization, multiple markers (e.g. genes, enzymes and structure proteins) must be analysed. These data can be used for a comparison with other stem cell types which have been shown to differentiate into the myogenic cell line (e.g. mesenchymal stem cells, hematopoietic stem cells or embryonic stem cells) in order to both discover the most promising stem cell type and optimize culture conditions for later clinical utilization.

\section{Acknowledgements}

The authors would like to thank Michael Collins for the great help with the manuscript and Petra Prohaska for the excellent technical assistance.

\section{References}

1. Beier JP, Stern-Straeter J, Foerster VT, Kneser U, Stark GB and Bach AD: Tissue engineering of injectable muscle: threedimensional myoblast-fibrin injection in the syngeneic rat animal model. Plast Reconstr Surg 118: 1113-1124, 2006.

2. Dusterhoft S and Pette D: Effects of electrically induced contractile activity on cultured embryonic chick breast muscle cells. Differentiation 44: 178-184, 1990.

3. Cronin KJ, Messina A, Knight KR, Cooper-White JJ, Stevens GW, Penington AJ and Morrison WA: New murine model of spontaneous autologous tissue engineering, combining an arteriovenous pedicle with matrix materials. Plast Reconstr Surg 113: 260-269, 2004

4. Bach AD, Beier JP, Stern-Straeter J and Horch RE: Skeletal muscle tissue engineering. J Cell Mol Med 8: 413-422, 2004.

5. Beier JP, Kneser U, Stern-Straeter J, Stark GB and Bach AD: Y chromosome detection of three-dimensional tissue-engineered skeletal muscle constructs in a syngeneic rat animal model. Cell Transplant 13: 45-53, 2004.

6. Messina A, Bortolotto SK, Cassell OC, Kelly J, Abberton KM and Morrison WA: Generation of a vascularized organoid using skeletal muscle as the inductive source. FASEB J 19: 1570-1572, 2005.

7. Levenberg S, Rouwkema J, Macdonald M, Garfein ES, Kohane DS, Darland DC, Marini R, van Blitterswijk CA, Mulligan RC, D'Amore PA and Langer R: Engineering vascularized skeletal muscle tissue. Nat Biotechnol 23: 879-884, 2005.

8. Stern-Straeter J, Riedel F, Bran G, Hormann K and Goessler UR: Advances in skeletal muscle tissue engineering. In Vivo 21: 435-444, 2007.

9. Blau HM and Webster C: Isolation and characterization of human muscle cells. Proc Natl Acad Sci USA 78: 5623-5627, 1981.

10. Perry RL and Rudnick MA: Molecular mechanisms regulating myogenic determination and differentiation. Front Biosci 5: D750-D767, 2000.

11. Brand-Saberi B: Genetic and epigenetic control of skeletal muscle development. Ann Anat 187: 199-207, 2005.

12. Skapek SX, Rhee J, Spicer DB and Lassar AB: Inhibition of myogenic differentiation in proliferating myoblasts by cyclin D1-dependent kinase. Science 267: 1022-1024, 1995. 
13. Sassoon DA: Myogenic regulatory factors: dissecting their role and regulation during vertebrate embryogenesis. Dev Biol 156: 11-23, 1993.

14. Hasty P, Bradley A, Morris JH, Edmondson DG, Venuti JM, Olson EN and Klein WH: Muscle deficiency and neonatal death in mice with a targeted mutation in the myogenin gene. Nature 364: 501-506, 1993.

15. Stern-Straeter J, Bach AD, Stangenberg L, Foerster VT, Horch RE, Stark GB and Beier JP: Impact of electrical stimulation on three-dimensional myoblast cultures - a real-time RT-PCR study. J Cell Mol Med 9: 883-892, 2005.

16. Machida S, Spangenburg EE and Booth FW: Primary rat muscle progenitor cells have decreased proliferation and myotube formation during passages. Cell Prolif 37: 267-277, 2004.

17. Bach AD, Stern-Straeter J, Beier JP, Bannasch H and Stark GB: Engineering of muscle tissue. Clin Plast Surg 30: 589-599, 2003.

18. Bach AD, Arkudas A, Tjiawi J, Polykandriotis E, Kneser U, Horch RE and Beier JP: A new approach to tissue engineering of vascularized skeletal muscle. J Cell Mol Med 10: 716-726, 2006.
19. Borschel GH, Dow DE, Dennis RG and Brown DL: Tissueengineered axially vascularized contractile skeletal muscle. Plast Reconstr Surg 117: 2235-2242, 2006.

20. Cronin EM, Thurmond FA, Bassel-Duby R, Williams RS, Wright WE, Nelson KD and Garner HR: Protein-coated poly(Llactic acid) fibers provide a substrate for differentiation of human skeletal muscle cells. J Biomed Mater Res A 69: 373$381,2004$.

21. Casar JC, Cabello-Verrugio C, Olguin H, Aldunate R, Inestrosa NC and Brandan E: Heparan sulfate proteoglycans are increased during skeletal muscle regeneration: requirement of syndecan-3 for successful fiber formation. J Cell Sci 117: 73-84, 2004.

22. Lev A and Holland PC: Developmental regulation of a secreted gelatin-binding protein during myogenesis in vitro. Biochem Cell Biol 65: 1031-1038, 1987.

23. Kitzmann M and Fernandez A: Crosstalk between cell cycle regulators and the myogenic factor MyoD in skeletal myoblasts. Cell Mol Life Sci 58: 571-579, 2001. 